\title{
Short-Duration Spaceflight Does Not Prolong QTc Intervals in Male Astronauts
}

\author{
Brett M. Mitchell, PhD, and Janice V. Meck, PhD
}

Although ventricular dysrhythmias are not increased during, and QTc intervals are not prolonged after, short-duration (5 to 16 days) spaceflights, QTc intervals have not previously been reported during these shorter flights. Holter monitor recordings, obtained in 11 male astronauts who flew on shuttle missions ranging from 5 to 10 days, showed that QTc intervals did not change significantly 10 days before launch, on 2 separate days of spaceflight, and 2 days after landing. Taken together, these data and our previous report show that QTc interval prolongation occurs sometime between the 9th and 30th days of spaceflight. (C) 2004 by Excerpta Medica, Inc.

(Am J Cardiol 2004;93:1051-1052)

Gard ardiac dysrhythmias have been reported on several occasions during long-duration ( 1 to 6 months) spaceflights, and some have impacted mission objectives. ${ }^{1-6}$ We and others have also reported that QTc intervals increase during long-duration spaceflights, indicating that ventricular repolarization processes may be altered. ${ }^{1,4}$ It is possible that the dysrhythmias and QTc prolongation are related. Although QTc intervals are not prolonged after, and ventricular dysrhythmias are not increased during, short-duration (5 to 16 days) spaceflights, in-flight QTc intervals have not been measured. Therefore, we used a retrospective analysis to test the hypothesis that QTc intervals are not prolonged during short-duration spaceflights.

Holter recordings taken from 11 male astronauts (mean age $35 \pm 5$ years) who flew on shuttle missions ranging from 5 to 10 days were retrospectively analyzed. Their heart rates and arterial pressure changes had been previously reported. ${ }^{7}$ Twenty-four-hour electrocardiographic data were collected 10 days before launch, twice in flight, and 2 days after landing. $\mathrm{PR}, \mathrm{RR}$, and QT intervals were measured in lead $\mathrm{V}_{1}$ for 10 cardiac cycles at $1,6,11$, and 16 hours after donning the Holter monitor. All time points analyzed were during normal activities (i.e., excluded exercise). Electrocardiographic analysis was performed as described previously. ${ }^{1}$ The corrected QT interval was calculated using Bazett's formula: QTc $=\mathrm{QT} /(\mathrm{RR})^{0.5} .8$ Data were checked for normalcy and expressed as

From the Universities Space Research Association, Houston, Texas; and the Space Life Sciences Research Laboratories, NASA/Johnson Space Center, Houston, Texas. This work was supported by grant CA00211FL from National Space Biomedical Research Institute, Houston, Texas. Dr. Meck's address is: Space Life Sciences Research Laboratories, Human Adaptation and Countermeasures Office, SK32/Johnson Space Center, NASA, Houston, Texas 77058. E-mail: jmeck@ems.jsc.nasa.gov. Manuscript received October 28, 2003; revised manuscript received and accepted December 24, 2003.
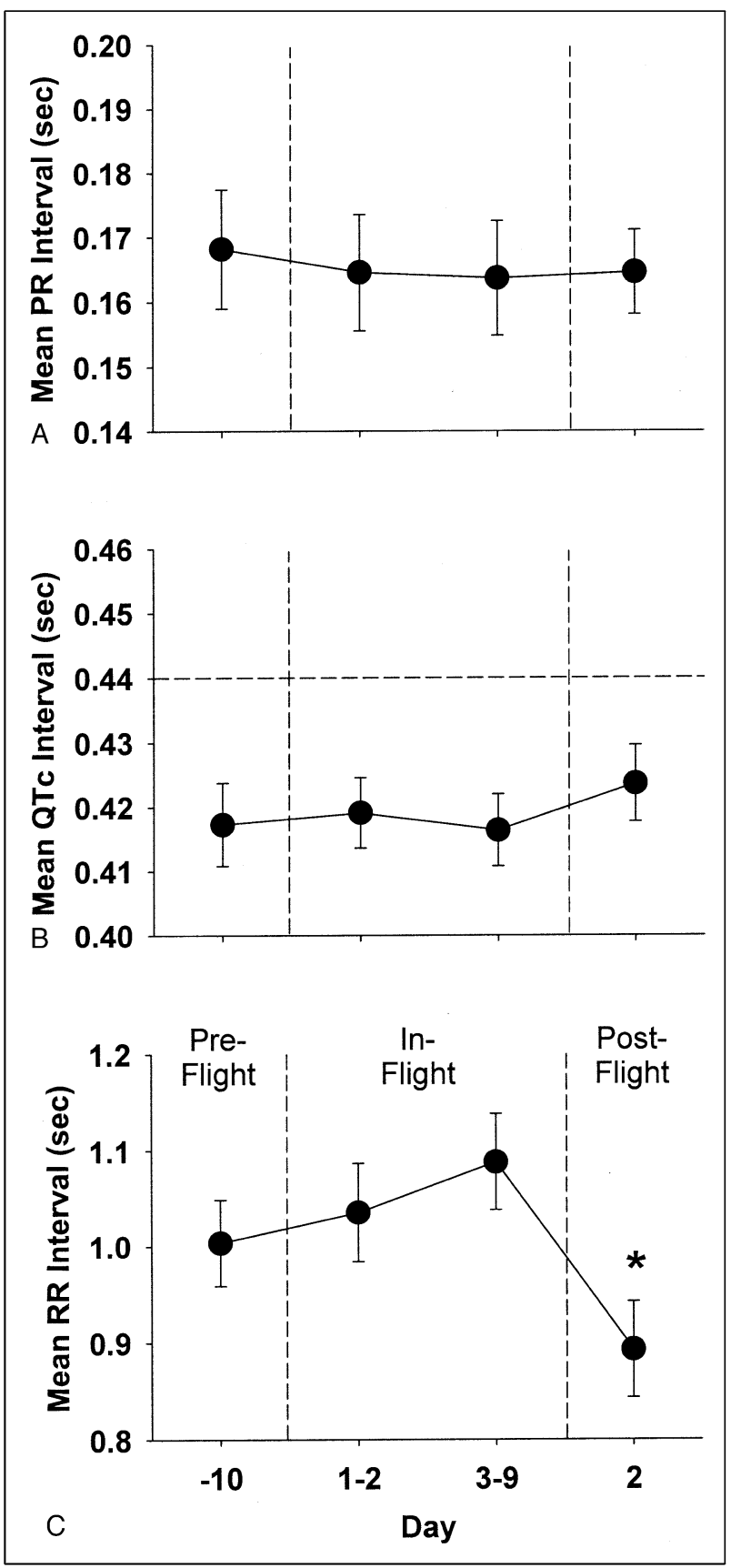

FIGURE 1. Mean (A) PR, (B) QTc, and (C) RR intervals from 24-hour Holter recordings for $11 \mathrm{crew}$ members at 10 days before launch (-10), on 2 in-flight days (1-2 and 3-9), and 2 days after landing (2). (B) Horizontal dotted line, clinical threshold of prolonged QTc interval (QTc $\geq 0.44$ second). ${ }^{*} \mathrm{p}<0.05$ compared with $-10,1-2$, and 3-9.

mean \pm SEM. An analysis of variance with repeated measures was used to examine differences within subjects 10 days before launch, on flight days, and 2 days 
after landing. Any significant main effects were further examined using the Tukey post hoc test. The significance level was set at $p<0.05$, and the power level achieved was $>0.80$.

Flights of $<10$ days did not alter PR or QTc intervals, and RR intervals decreased significantly 2 days after landing compared with previous days (Figure 1). Six astronauts had increased and 5 had decreased QTc intervals during spaceflight, suggesting individual variability. The largest change was 0.02 second. Interestingly, 3 astronauts had QTc intervals $\geq 0.44$ second before spaceflight. Of these 3, the QTc interval increased during spaceflight in 1 and decreased in the others. Mean QTc intervals from the 3 long-duration astronauts reported previously ${ }^{1}$ were identical before spaceflight compared with our present study $(0.41 \pm 0.01$ second $)$ but increased significantly after 30 days $(0.46 \pm 0.02$ second $)$, 60 days $(0.48 \pm 0.02$ second $)$, and 90 days $(0.49 \pm 0.02$ second) of spaceflight.

In this study, QTc intervals did not increase significantly during the first 9 days of spaceflight. Based on our previous work demonstrating prolongation of QTc intervals in astronauts on long-duration spaceflights, we speculate that QTc interval prolongation occurs sometime between 9 and 30 days of spaceflight. Perhaps after the longer exposure to a reduced workload in flight, the heart begins to remodel. Interestingly, this group of astronauts had longer QTc intervals than a previously reported group of astronauts who underwent short-duration spaceflight, ${ }^{1}$ and several had QTc intervals that were at or near the clinical threshold of prolonged QTc (QTc $\geq 0.44$ second) before spaceflight. Future studies should examine this time-dependent effect of spaceflight on QTc intervals.

Acknowledgment: We express our appreciation to the astronauts who volunteered for this study and to L. Christine Ribeiro, MS, for assistance with data analysis.

1. D'Aunno DS, Dougherty AH, DeBlock HF, Meck JV. Effect of short- and long-duration spaceflight on QTc intervals in healthy astronauts. Am J Cardiol 2003;91:494-497.

2. Rossum AC, Ziegler MG, Meck JV. Effect of spaceflight on cardiovascular responses to upright posture in a 77-year-old astronaut. Am J Cardiol 2001;88: 1335-1337.

3. Fritsch-Yelle JM, Leuenberger UA, D'Aunno DS, Rossum AC, Brown TE, Wood ML, Josephson ME, Goldberger AL. An episode of ventricular tachycardia during long-duration spaceflight. Am J Cardiol 1998;81:1391-1392.

4. Golubchikova ZA, Alferova IV, Liamin VR, Turchaninova VF. Dynamics of some electrocardiographic parameters in cosmonauts during long-term Mir mission. Aviakosm Ekolog Med 2003;37:41-45.

5. Hoffler GW, Wolthuis RA, Johnson RL. Apollo space crew cardiovascular evaluations. Aerosp Med 1974;45:807-820.

6. Romanov EM, Artamonova NP, Golubchikova ZA, Zavadovskii AF, Korotaev MM. Results of long-term electrocardiographic examinations of cosmonauts. Kosm Biol Aviakosm Med 1987;21:10-14.

7. Fritsch-Yelle JM, Charles JB, Jones MM, Wood ML. Microgravity decreases heart rate and arterial pressure in humans. J Appl Physiol 1996;80:910-914.

8. Bazett HC. An analysis of the time relation of electrocardiograms. Heart 1920;7:353-370.

\title{
Bradycardia During Therapy for Multiple Myeloma With Thalidomide
}

\author{
Ibrahim Elias Fahdi, MD, Venkat Gaddam, MD, Jorge F. Saucedo, MD, \\ Channarayapatna V. Kishan, MD, Keyur Vyas, MD, Matthew G. Deneke, MD, \\ Hani Razek, MD, Brett Thorn, MS, Joseph K. Bissett, MD, Elias Anaisse, MD, \\ Bart Barlogie, MD, and Jawahar L. Mehta, MD, PhD
}

\begin{abstract}
We studied the medical records of 96 patients who received thalidomide and 104 patients who made up a control group. We found that $53 \%$ of patients $(52$ patients) using thalidomide had a heart rate of $<60$ beats/min at some point during follow-up and $19 \%$ of thalidomide patients (10 patients) developed symptom-related bradycardia. Reducing the thalidomide dose appeared to alleviate symptoms in most patients. () 2004 by Excerpta Medica, Inc.
\end{abstract}

(Am J Cardiol 2004;93:1052-1055)

\footnotetext{
From the Division of Cardiovascular Medicine, the Department of Internal Medicine, and the Multiple Myeloma Center, University of Arkansas for Medical Sciences, Little Rock, Arkansas; and the University of Oklahoma Health Sciences Center, Oklahoma City, Oklahoma. Dr. Mehta's address is: Division of Cardiovascular Medicine, University of Arkansas for Medical Sciences, 4301 West Markham Street, \#532, Little Rock, Arkansas 72205. E-mail: mehtaj|@uams.edu. Manuscript received August 27, 2003; revised manuscript received and accepted December 18, 2003.
}

$\mathbf{T}$ halidomide ( $\alpha$-N-phthalimidoglutarimide) was initially manufactured in West Germany and was marketed as an antiemetic and sedative until 1960. Thalidomide functions as a potent immunosuppressive and antiangiogenic agent ${ }^{1-3}$ by inhibiting the phagocytic ability of inflammatory cells and the production of cytokines, such as tumor necrosis factor- $\alpha$ (TNF$\alpha$ ). It has been shown to be effective in the treatment of inflammatory diseases, ${ }^{4-6}$ in conditions associated with human immunodeficiency virus (HIV) infection, ${ }^{7}$ and in various cancers. ${ }^{8-10}$ Vogelsang et al ${ }^{11}$ showed improved survival in patients with refractory chronic graft-versus-host reaction who received thalidomide. One-third of patients with advanced and refractory multiple myeloma, in whom salvage therapy failed after single and even tandem autotransplants, responded markedly to this drug. ${ }^{10,12}$ Thalidomide represents only the third independently active compound in the treatment of myeloma since the introduction of melphalan and prednisone 4 decades ago. Thalido- 